\title{
Coupling effects of heterogeneity and stress fluctuation on rupture
}

\author{
X.H. Zhang ${ }^{\text {a }}$, F. Rong ${ }^{\text {a }}$, Z.K. Jia ${ }^{\text {a }}$, F.J. Ke ${ }^{\text {a,b }}$, M.F. Xia ${ }^{\text {a,c }}$, Y.L. Bai ${ }^{\text {a,* }}$ \\ a State Key Laboratory of Non-linear Mechanics (LNM), Institute of Mechanics, Chinese Academy of Sciences, \\ Beijing 100080, China \\ ${ }^{\mathrm{b}}$ Department of Applied Physics, Beijing University of Aeronautics and Astronautics, Beijing 100083, China \\ ${ }^{\mathrm{c}}$ Department of Physics, Peking University, Beijing 100871, China
}

\begin{abstract}
The coupling of mesoscopic strength distribution and stress fluctuation on damage evolution and rupture are examined. The numerical simulations show that there is only weak stress fluctuation at the initial damage stage when the mean field approximation is in effect. As the damage fraction becomes larger than the threshold value, the fluctuation is amplified significantly, and damage localization appears. The coupling between stress fluctuation, disordered heterogeneity and the damage localization may play an essential role in catastrophic rupture.
\end{abstract}

(C) 2003 Elsevier Ltd. All rights reserved.

Keywords: Heterogeneity; Stress fluctuation; Damage localization; Catastrophic rupture

\section{Introduction}

Media with mesoscopic heterogeneity, like rock, are important in practice but their properties appear to be diversified. Mathematically speaking, mesoscopic heterogeneity implies the dependence of macroscopic behaviors on mesoscopic coordinates. In the other hand, engineering practice however prohibits the macroscopic constitutive coefficient to depend on mesoscopic coordinates since this would render the problem inaccessible. Statistical distribution functions would be a better choice than depending on the meso-coordinate.

\footnotetext{
${ }^{*}$ Corresponding author.

E-mail address: baiyl@lnm.imech.ac.cn (Y.L. Bai).
}

The important statistical descriptions are:

- The statistical distributions of mesoscopic variables may involved the distribution function of material properties (elastic parameters, mesoscopic strength, etc.) and the distribution function of stresses, etc. These distribution functions may become time-dependent owing to damage evolution. The corresponding macroscopic variables can be calculated from the statistical average of the relevant distribution functions, e.g., the damage fraction could be related to the distribution function of mesoscopic strength and the loading history.

- The statistical average of joint probability mesoscopic variables can lead to the correlation function between microdamages, stresses, etc. 
Although the effect of heterogeneity on stressstrain curve in the media have been discussed by previous authors [1-5], the usual approaches are based on mean field approximation. That is to say, the stress on a macroscopic sample is assumed to be uniform, to permit a simple macroscopic constitutive description of the material. However, it is found that the behavior near the rupture of a sample obtained by the mean field approximation may not be in agreement with observations. This is mainly because the stress fluctuation becomes stronger and stronger as rupture is approached in the heterogeneous media. Coupling effects of the stress fluctuation and the mesoscopic heterogeneity of the material is essential in catastrophic rupture.

In this paper, emphasis is placed on the stress distribution function and its effects on rupture. Numerical simulations for a network model under uniaxial tensile loading accounted for by considering the stress fluctuation. The rupture of networks occurs significantly earlier than that based on mean field approximation. To interpret the difference, the coupling effect between stress fluctuation and heterogeneity of mesoscopic strength should be taken into account. Through stress fluctuation statistics of network model, it is found that at the initial damage stage, relative variance of strain energy increases linearly with the damage fraction; when the damage fraction becomes larger, values of relative strain energy variance increase quickly with increasing damage fraction.

\section{Mean field approximation}

Although the results of mean field approximation are not in agreement with the observations near rupture, they may give an important clue to understand the mechanism of rupture. Hence, a brief review of the mean field approximation will be given. Since rupture is much more sensitive to mesoscopic strength than elastic modulus, an elasticbrittle model is adopted to obtain a mesoscopic elastic modulus $E_{0}$ but various mesoscopic strength can be depicted by the distribution function, $h\left(\sigma_{\mathrm{c}}\right)$, which is assumed to have a Weibull distribution function with two parameters. They are the scale parameter $\eta$ and Weibull modulus $m$ (shape para- meter) [6]. Provided are the normalized stress $\sigma=$ stress $/ \eta$ and normalized strain $\varepsilon=$ strain $*$ $E_{0} / \eta$ such that $\sigma_{\mathrm{c}}=\varepsilon_{\mathrm{c}}$ for any elastic-brittle mesoscopic elements of a macroscopic sample of the heterogeneous medium. Hence, the Weibull distribution of normalized strength $\sigma_{\mathrm{c}}$ is

$h\left(\sigma_{\mathrm{c}}\right)=h\left(\varepsilon_{\mathrm{c}}\right)=m \cdot \sigma_{\mathrm{c}}^{m-1} \cdot \exp \left(-\sigma_{\mathrm{c}}^{m}\right)$.

The smaller the Weibull modulus $m$ is, the more diverse the meso-strength is. That is to say, the more heterogeneous the medium is. Based on the mean field approximation, the corresponding constitutive equation is

$\sigma(\varepsilon)=\varepsilon \int_{\varepsilon}^{\infty} h\left(\varepsilon_{\mathrm{c}}\right) \cdot \mathrm{d} \varepsilon_{\mathrm{c}}=\varepsilon \cdot \exp \left(-\varepsilon^{m}\right)$,

where $D$ is the damage fraction determined by

$D=\int_{0}^{\varepsilon} h\left(\varepsilon_{\mathrm{c}}\right) \cdot \mathrm{d} \varepsilon_{\mathrm{c}}=1-\exp \left(-\varepsilon^{m}\right)$.

For this heterogeneous elastic-brittle model, there is a sequence of events, among which damage localization and catastrophic rupture may be the most interesting.

Physically, damage localization implies the emergence of macroscopic inhomogeneity, i.e. a prelude to localized rupture. The geometrical definition of damage localization $[7,9]$ is

$\frac{\frac{\partial}{\partial T}\left(\frac{\partial D}{\partial X}\right)}{\frac{\partial D}{\partial X}} \geqslant \frac{\frac{\partial D}{\partial T}}{D}$.

For the medium with Weibull distribution, the critical conditions for damage localization can be derived as

$$
\begin{aligned}
& (m-1) \cdot D_{\mathrm{L}}+m \cdot \ln \left(1-D_{\mathrm{L}}\right) \cdot\left[m \cdot\left(1-D_{\mathrm{L}}\right)\right. \\
& \left.\cdot \ln \left(1-D_{\mathrm{L}}\right)+1-2 D_{\mathrm{L}}\right]=0,
\end{aligned}
$$

where $D_{\mathrm{L}}$ is the critical damage for damage localization. As $D \geqslant D_{\mathrm{L}}$, the fluctuations of the damage fraction and the stress will be amplified and leads to appearance of damage localization. It is interest to notice that the criterion of damage localization is derived based on mean field approximation, but the criterion really corresponds to the condition where the mean field approximation tends to be inapplicable. 
The critical condition for the self-sustained catastrophic rupture is that the increase of input work $\Delta W$ is no longer positive, i.e.,

$$
\begin{aligned}
\Delta W & =F \cdot\left(\Delta u_{\mathrm{s}}+\Delta u_{\mathrm{m}}\right) \\
& =F \cdot \Delta F \cdot\left(K_{\mathrm{s}}^{-1}+K_{\mathrm{m}}^{-1}\right) \leqslant 0,
\end{aligned}
$$

where $F, \Delta u$ and $K$ are load, the increments of displacement and stiffness, respectively, and subscripts 's' and ' $m$ ' refer to the sample and testing machine, respectively. Suppose $K_{\mathrm{m}}=k K_{\mathrm{s} 0}, K_{\mathrm{s} 0}$ is the initial stiffness of the sample, the instability condition for catastrophic rupture (6) can be expressed as $\frac{\mathrm{d} \sigma}{\mathrm{d} \varepsilon} \leqslant-k$

The case driven by external load is corresponding to $k=0$, the catastrophic rupture appears at the maximum point of the constitutive curve. The case driven by boundary displacement is corresponding to $k \rightarrow \infty$, the failure of the sample shows continuous damage accumulation and does not display catastrophe (see Fig. 1(a)). The case of $k=1$ is shown in Fig. 1(b).

Fig. 2 gives an example of the variation of stress and damage with increasing strain $(m=5)$. The

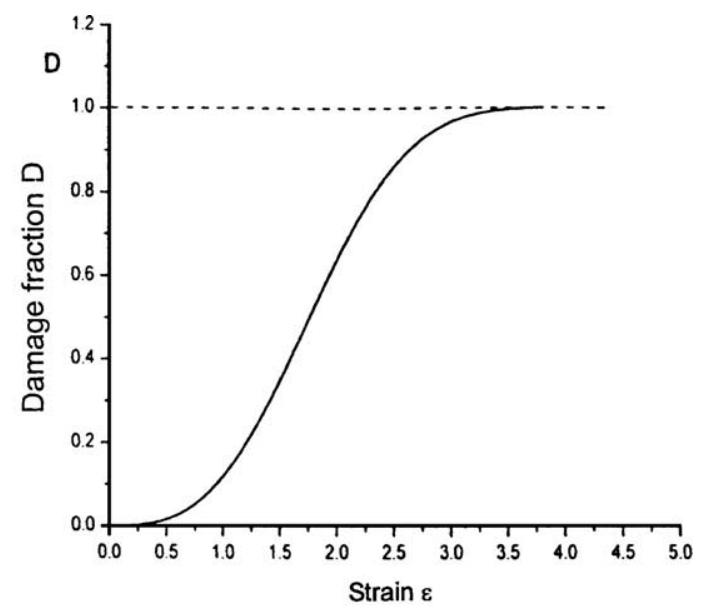

(a)
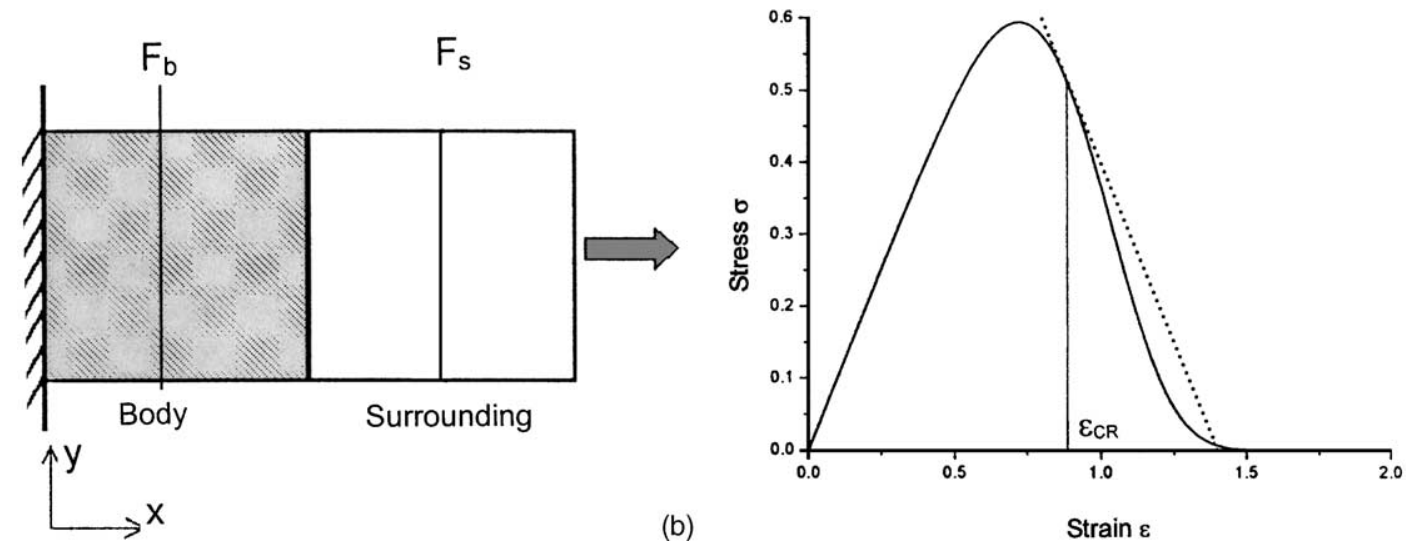

(b)

Strain $\varepsilon$

Fig. 1. (a) Continuous damage evolution. $D$ denotes the damage fraction, $\varepsilon=u / L$, where $\varepsilon$ is the strain, $L$ is the length of the sample and $u$ is the boundary displacement. Quasi-static loading is assumed. $h(\varepsilon)$ is Weibull distribution function with modulus $m=3$. (b) The schematic of the specimen and surrounding (machine) (left) and the curve of stress vs. strain for $k=1$ and $m=5$ in MF approximation (right). $\varepsilon_{\mathrm{cr}}$ indicates catastrophic rupture, where the tangent of the stress-strain curve becomes the negative of the stiffness of the machine (the straight line). 


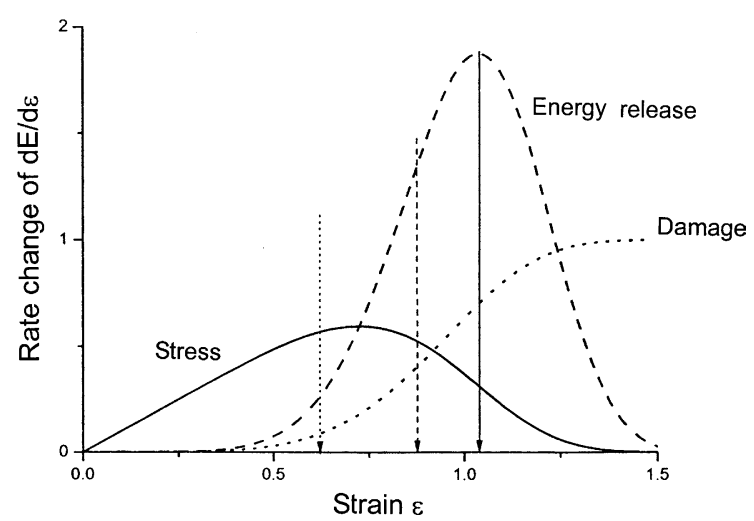

Fig. 2. The stress-strain curve (thick solid line), gradual energy release rate $\frac{\mathrm{d} E}{\mathrm{~d} \varepsilon}$ vs. strain (thick dashed) and damage vs. strain (thick dotted) for the model with $m=5$ based on the mean field (MF) approximation. The vertical lines (from left to right) indicate the strains where damage localization (dotted), catastrophic rupture $(k=1$, dashed) and both maximum energy release and minimum slope of stress-strain curve (solid) occur.

figure clearly demonstrates that damage localization, maximum stress and catastrophic rupture occur successively with increasing deformation. Moreover, for any cases (any $m$ values) damage localization always appears before maximum stress and catastrophic rupture. In a broad sense, damage localization may be adopted as a very early warning of rupture.

In some cases the results obtained by the mean field approximation are not in agreement with observations, because of stress fluctuation in the sample [8].

\section{Characteristics of stress fluctuation and effects of stress fluctuation}

The deviation of stress in the sample from the mean field approximation may be enhanced owing to damage-induced stress re-distribution. Therefore, in a real system the coupling between stress fluctuation and heterogeneous mesoscopic strength may play an important role.

Numerical simulations were performed with a two-dimensional network model $[10,11]$. The network consists of straight bars of the same length and the same elastic modulus but different tensile strength. Each bar deforms elastically until its own breaking threshold, i.e. tensile strength is reached. Similar to the previous analytical model, the breaking thresholds of all bars of the network follow the Weibull distribution, Eq. (1). The spatial distribution of the breaking thresholds in the network, however, is random and statistically uniform. The bars support tensile or compressive loads only and six bars are hinged at a joint, see Fig. 3. In the calculations, networks consisting of $40 \times 40$ and $60 \times 60$ bars are adopted. The boundary conditions are shown in Fig. 3. On the lefthand side of the network, all joints of the bars are hinged to a straight fixed solid wall. On the right-hand side of the network, a similar network with the same elastic modulus but without breaking is attached as a mimic testing machine. External uniaxial tensile loading is applied on the extreme right of the surrounding, Fig. 3. Though the load applied to the sample is uniaxial, the bars in the network may sustain different stresses owing to different orientations and mesoscopic strength. More importantly, since the bars have different breaking thresholds and then fail individually according to its local stress, then stress re-distribution will appear in the two-dimensional network.

The two-dimensional simulations under tension in Fig. 4 correspond to the early stage of deformation and damage. There is a good consistency between the simulations and the mean field approximation for either Weibull modulus $m=5$ or 2. But, when there are some deviations in stressstrain curves from the mean field approximations, sudden rupture occurs. The rupture appears earlier than the mean field predictions for catastrophic rupture, and even earlier than the predictive maximum stress. However, the unloading path in all these simulations is exactly the same as those predicted by the mean field approximation. The unloading slope is equal to the negative stiffness of the mimic testing machine. One hint for the interpretation of the immature rupture in simulations is that all ruptures in simulations occur beyond damage localization, Fig. 4. Recall that the assumptions made in the analysis of catastrophic rupture in terms of the mean field approximation, i.e. the assumption of uniform spatial stress and damage distributions may be improper, since the 

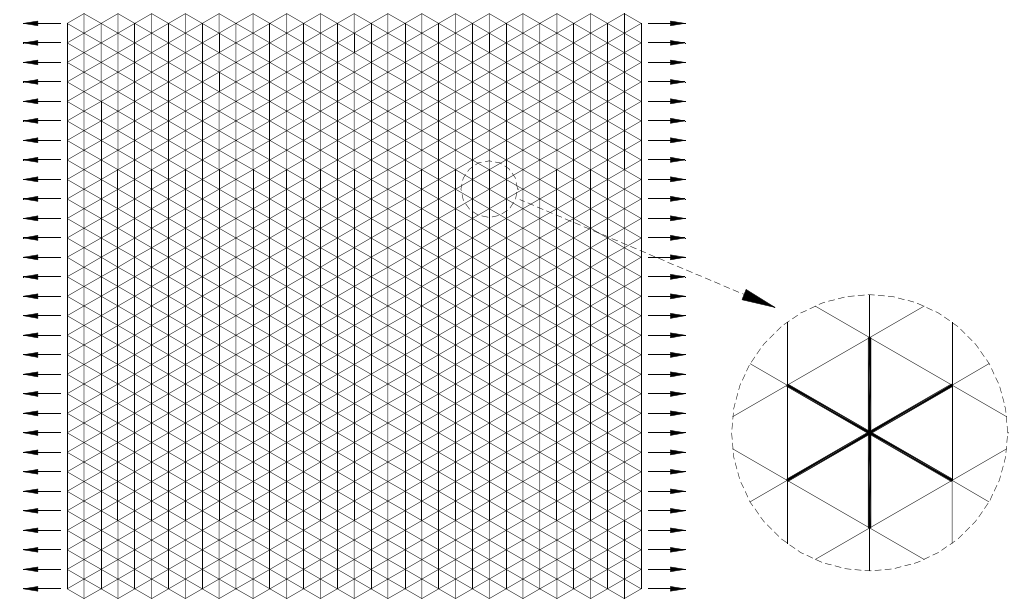

Fig. 3. Sketch of network model. The network consists of straight bars of the same length. The spatial distribution of breaking thresholds of the bars in the network is random and statistically uniform. The bars support tensile or compressive loads only and six bars are hinged at a joint.
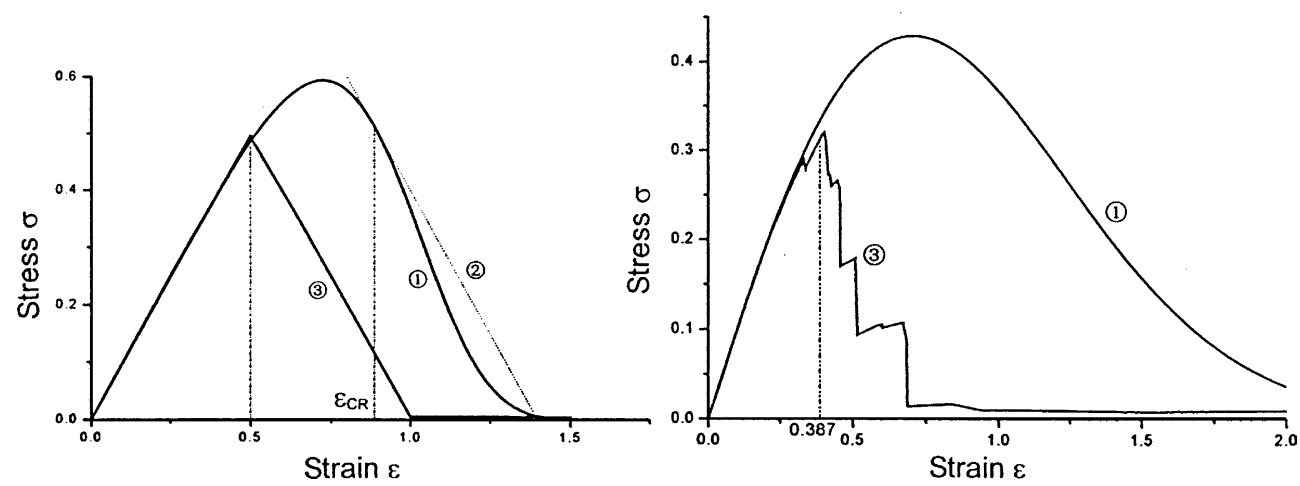

Fig. 4. The curves of stress vs. strain coupled with surrounding for Weibull distribution with moduli $m=5$ (left) and $m=2$ (right), respectively. In the diagram, curve (1) represents the result of the mean field model with infinite surrounding stiffness. Curve (2) shows the jump unloading after catastrophic rupture (marked by a vertical dashed line labelled as $\varepsilon_{\mathrm{cr}}$ ) owing to finite surrounding stiffness, Eq. (7). Curve (3) is the result of numerical simulation of network driven by the surrounding with finite stiffness. The two vertical dashed lines labelled by 0.5 (left) and 0.387 (right) indicate damage localization $\left(\varepsilon_{\mathrm{L}}=0.501\right.$ for $m=5$ and $\varepsilon_{\mathrm{L}}=0.387$ for $m=2$ ). Notice that the rupture occurs beyond the appearance of damage localization in the simulations. But in the right diagram, there are only curves (1) and (3), and there is no curve (2) in the case of $m=2$, because there is no point on curve (1) satisfying the condition for catastrophic rupture, Eq. (7).

damage localization implies uneven spatial damage. The uneven damage localization induces more severe uneven spatial stress re-distribution, which can enhance local damage accumulation.

The stress patterns in Fig. 5 show that the whole process may be divided into three stages. At the initial stage, several small cracks occur and the stress-strain curve remains nearly linear. With increasing damage, high stress regions may shift from one part to another very swiftly. This means that severe stress adjustment occurs. At the final stage, a main crack forms, and high stress mainly adheres to the large crack tip. From the simulations, it can be seen that the stress re-distribution due to heterogeneity plays a very significant role in rupture.

Actually, the network simulations show clear stress concentration and stress screening effect when 


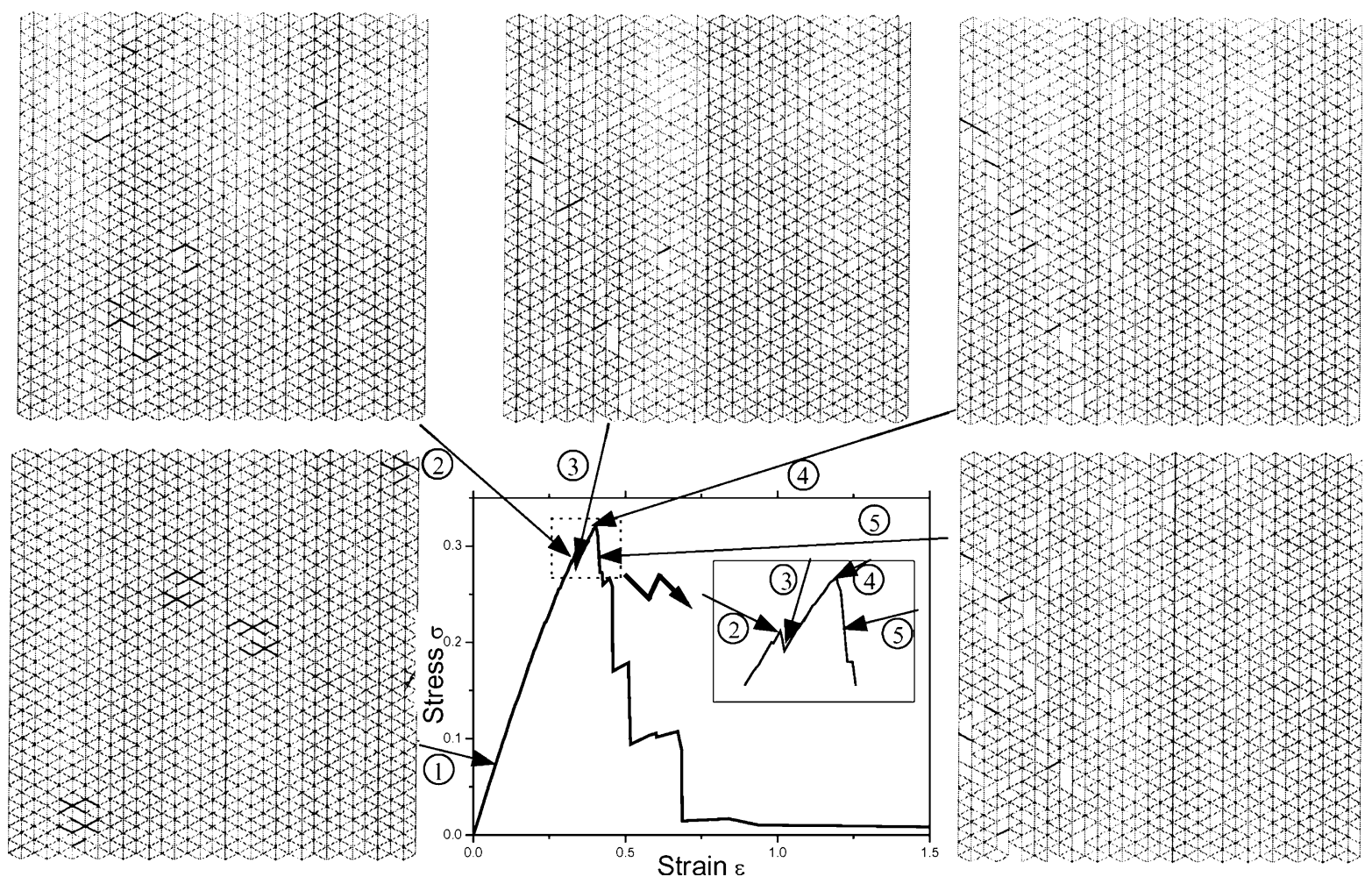

Fig. 5. Stress re-distribution and damage patterns in network simulation (for Weibull modulus $m=2$ ) and the corresponding stress vs. strain curve. The bars in the network are colored with 10 levels of darkness according to their stress, bold black indicates the highest stress and white vacancy means bar broken. From the insets, one can notice clear damage localization and stress re-distribution ahead of rupture.

some elastic bars fail during the loading process. Now, consider the characteristics of stress fluctuation in the rupture process, the network model is based on the equivalence of strain energy stored in a unit element [12]. Statistics on strain energy will be made to investigate the stress fluctuations indirectly.

The relative variance of strain energy $\left(\frac{\delta E}{E}\right)^{2}$ is defined as square of the standard deviation of strain energy divided by average strain energy of a unit element. Figs. 6 and 7 show the relation between the relative variance of strain energy ( $Y$ axis) and the damage fraction ( $X$ axis). The black solid line is the results of a percolation model. In the percolation model the broken bar is simply selected at random for each step. So, the broken bars are statistically uniformly distributed. The results of percolation model are compared with those obtained by the network model. Ten network samples are computed with the same Weibull modulus. It is found that:

- During the initial damage period (damage fraction is less than $2 \%$ ), relative variance of strain energy increases linearly with the damage fraction. After comparing the results of network model and percolation model, it can be concluded that the fluctuation at this period is mainly caused by randomly distributed damage. And the interaction among damage can be neglected at this stage, Fig. 6.

- When the damage fraction becomes larger, Fig. 7 the values of relative strain energy variance increase quickly with increasing damage fraction and they are much larger than that of percolation model. When damage is becoming localized, the interaction of microdamage leads to a nonlinear increase of the strain energy variance. 


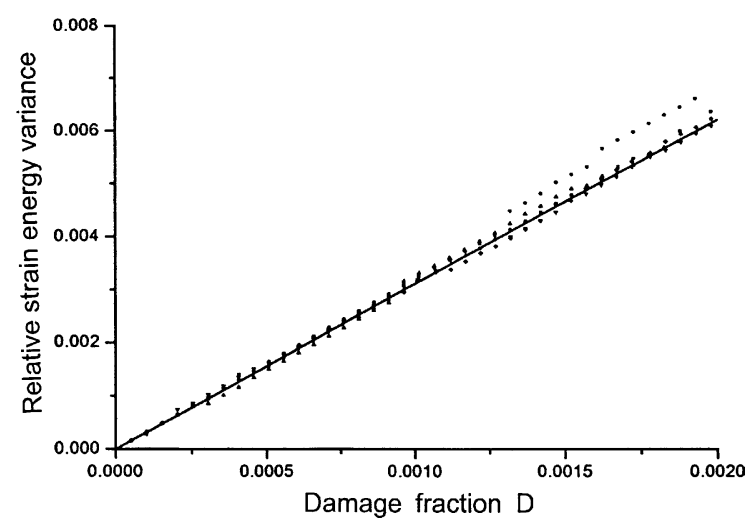

Fig. 6. The relative strain energy variance vs. damage fraction for the network model (dotted line) with $m=3$ and percolation model (black solid line).

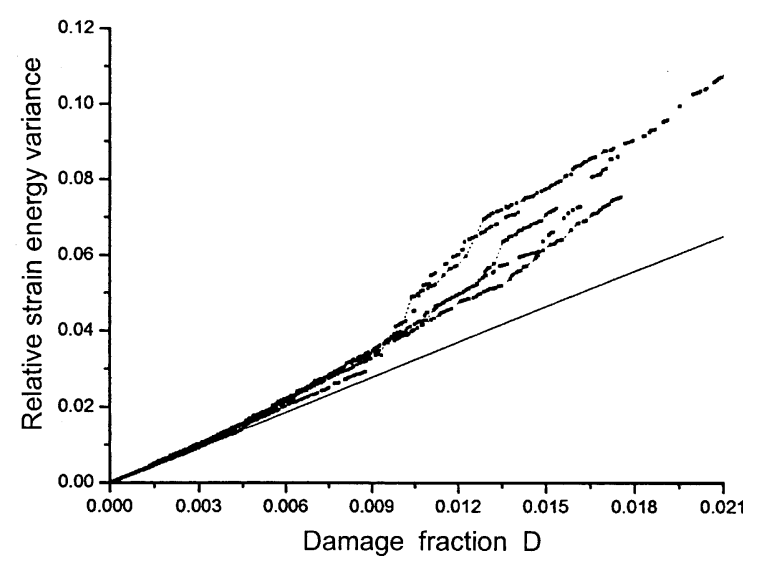

Fig. 7. The relative strain energy variance vs. damage fraction for the network model with $m=3$ (dotted line) and percolation model (black solid line).

It is also found that the values of the relative strain variance are obviously different from sample to sample. It seems that it is the stress fluctuation that leads to the catastrophe prior to the maximum stress given by MF model and the sample-specific behavior.

\section{Damage localization on catastrophic rupture}

The fully developed stress fluctuation leads to appearance of damage localization, which may affect the rupture behavior significantly. As mentioned above, the global mean field approximation for

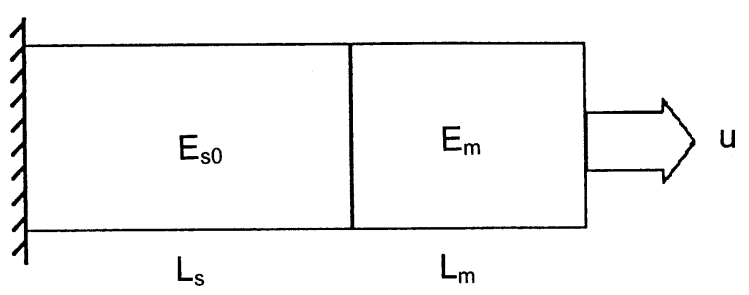

Fig. 8. Sketch of the simplified model.

failure of the sample driven by boundary displacement $u$ quasi-statically shows continuous damage accumulation and does not display catastrophic behavior. However, the transition of evolution mode from homogeneous damage to damage localization [3,4] may result in the appearance of catastrophic transition.

In order to reveal the mechanism of damage localization induced catastrophe, consider a simplified model of damage localization. Suppose that the sample divided into two (or more) parts: a heavily damaged one attached to an elastic one shown in Fig. 8.

There are two parts in the sample: an elastic part with length $L_{\mathrm{m}}$ and constant elastic modulus $E_{\mathrm{m}}$; a damaged part with length $L_{\mathrm{s}}$ and initial elastic modulus $E_{\mathrm{s} 0}$, and the strength threshold of mesoscopic units in the damage part follows Weibull distribution function with modulus $m$. Use the local mean field approximation to the sample, i.e., the mean field approximation is used to each part, separately. Denote the strain for two parts by $\varepsilon_{\mathrm{s}}$ and $\varepsilon_{\mathrm{m}}$, separately. This gives

$u=L_{\mathrm{s}} \varepsilon_{\mathrm{s}}+L_{\mathrm{m}} \varepsilon_{\mathrm{m}}$.

The displacement $u$ is expressed by using the unit corresponding to that of the strain $\varepsilon$. The balance condition is expressed by

$E_{\mathrm{m}} \varepsilon_{\mathrm{m}}=E_{\mathrm{s} 0}\left(1-D\left(\varepsilon_{\mathrm{s}}\right)\right) \varepsilon_{\mathrm{s}}$.

The damage fraction is determined by

$D\left(\varepsilon_{\mathrm{s}}\right)=\int_{0}^{\varepsilon_{\mathrm{s}}} h\left(\varepsilon_{\mathrm{c}}\right) \mathrm{d} \varepsilon_{\mathrm{c}}$.

The catastrophic transition appears at

$\frac{\mathrm{d} D}{\mathrm{~d} u}=\frac{\frac{\mathrm{d} D}{\mathrm{~d} \varepsilon_{\mathrm{s}}}}{\frac{\mathrm{d} u}{\mathrm{~d} \varepsilon_{\mathrm{s}}}}=\infty \quad$ or $\quad \frac{\mathrm{d} u}{\mathrm{~d} \varepsilon_{\mathrm{s}}}=0$. 
From Eqs. (8), (9) and (11), this provides the condition

$\frac{1}{k}-g\left(\varepsilon_{\mathrm{s}}\right)=0$,

where

$k=\frac{E_{\mathrm{s} 0} L_{\mathrm{b}}}{E_{\mathrm{b}} L_{\mathrm{s}}}$

and

$g\left(\varepsilon_{\mathrm{s}}\right)=-1+D\left(\varepsilon_{\mathrm{s}}\right)+\varepsilon_{\mathrm{s}} h\left(\varepsilon_{\mathrm{s}}\right)$.

The equation is solvable only for the case of

$k>\frac{1}{g_{\text {Max }}}$.

Denote the solution of Eq. (12) by $\varepsilon_{\mathrm{sc}}$ (or $u_{\mathrm{c}}$, or $D_{\mathrm{c}}=D\left(\varepsilon_{\mathrm{sc}}\right)$ ). The catastrophe appears at $u=u_{\mathrm{c}}$ (or $\varepsilon_{\mathrm{s}}=\varepsilon_{\mathrm{sc}}$ ), when the condition (15) is satisfied.

As the threshold follows Weibull distribution function with modulus $m$, the condition for appearance of catastrophe becomes

$k>\frac{\exp \left(1+\frac{1}{m}\right)}{m} \equiv k_{\mathrm{c}}(m)$

or

$\beta=\frac{k}{k_{\mathrm{c}}(m)}>1$.

The catastrophe transition point is at $\varepsilon_{\mathrm{sc}}\left(\right.$ or $\left.D_{\mathrm{c}}\right)$, which satisfies

$\frac{1}{k}+\exp \left(-\varepsilon_{\mathrm{sc}}^{m}\right)-m \varepsilon_{\mathrm{sc}}^{m} \exp \left(-\varepsilon_{\mathrm{sc}}^{m}\right)=0$

or

$\frac{1}{k}+1-D_{\mathrm{c}}+m\left(1-D_{\mathrm{c}}\right) \ln \left(1-D_{\mathrm{c}}\right)=0$.

Fig. 9 shows the damage evolution for the case with $m=3$ and $\beta=1.186>1\left(k_{\mathrm{c}}(3)=1.264555965\right.$, $k=1.5)$. The catastrophe transition depends on mesoscopic heterogeneity and damage evolution, especially the scale of damage localization the local damage fraction. The critical damage fraction $D_{\mathrm{c}}$ is dependent on $m$ and $k$ in above-mentioned example. However, the damage localization can be the

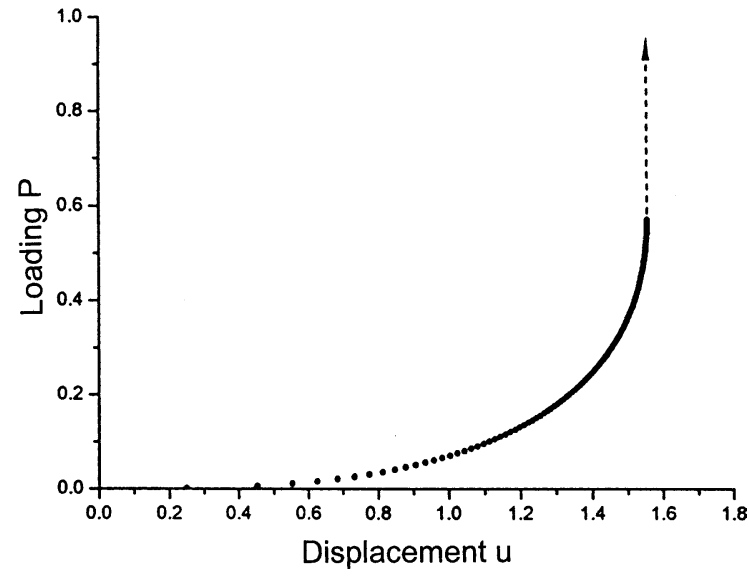

Fig. 9. Damage localization induced catastrophe transition. Quasi-static loading is assumed. $h(\varepsilon)$ is Weibull distribution function with modulus $m=3$ and $\beta=1.186$.

result of enhancement of fluctuations from mesoscopic level to macroscopic level. So the damage localization may display sample-specificity since it is sensitive to the details on mesoscopic scale. Then the catastrophe transition may also present macroscopic uncertainty, which leads to the difficulty in rupture prediction because the details on mesoscopic scale are difficult to be identified.

\section{Discussion}

The coupling effects of mesoscopic strength distribution and stress fluctuations on damage evolution and rupture are examined. The mean field approximation of a heterogeneous brittle model demonstrates a sequence of characteristic events, which includes damage localization, maximum stress and catastrophic rupture, etc. Damage localization always appears ahead of maximum stress and its dependence on heterogeneity could be simply calculated by means of the mean field approximation.

Though the mean field approximation can properly indicate the appearance of damage localization. It does imply that the mean field approximation tends to be lacking. The numerical simulations of heterogeneous networks under tension show that rupture may occur earlier than 
that predicted based on mean field approximation. Data analysis demonstrates that this may result from stress fluctuation in the sample. Therefore, stress fluctuation and its interaction with microdamage evolution should be taken into account in the discussion of rupture.

The statistics of stress fluctuation in the numerical simulations show that at the initial damage stage, relative variance of strain energy increases linearly with the damage fraction. The fluctuations in this period are mainly caused by randomly distributed damage. When the damage fraction becomes larger, the relative strain energy variance increases quickly with increasing damage fraction. This may significantly affect rupture.

The damage localization resulting from the coupling effects between stress fluctuation and disordered heterogeneity presents uncertainty or sample-specificity. This leads to sample-specificity of catastrophic rupture. The sample-specific catastrophe, a typical complexity in heterogeneous media, is the main origin of the difficulty of rupture prediction.

\section{Acknowledgements}

This research is funded by the National Natural Science Foundation of China (NSFC No. 10232050, 10372012 and No. 10172084) and Major State Research Project "Nonlinear Science" G2000077305.

\section{References}

[1] J.C. Jarger, N.G.W. Cook, Fundamentals of Rock Mechanics, Chapman and Hall, London, 1979.

[2] A.D.E.S. Jayatilaka, Fracture of Engineering Brittle Materials, Applied Sciences Publishers, London, 1979.

[3] L.S. Costin, Time-dependent deformation and failure, in: B.K. Atkinson (Ed.), Fracture Mechanics of Rock, Academic Press, 1987.

[4] C.A. Tang, Catastrophe in Rock Unstable Failure, Coal Industry Press, Beijing, 1993 (in Chinese).

[5] D. Krajcinovic, Damage Mechanics, Elsevier Science, Amsterdam, 1996.

[6] W. Weibull, A statistical distribution function of wide applicability, ASME Trans. J. Appl. Mech. 18 (1951) 293297.

[7] H.L. Li, Y.L. Bai, M.F. Xia, F.J. Ke, Damage localization as possible mechanism underlying earthquakes, PAGEOPH 157 (2000) 1929-1943.

[8] X.H. Zhang, X.H. Xu, M.F. Xia, F.J. Ke, Y.L. Bai, Critical sensitivity in a driven non-linear system, PAGEOPH, 161(9/10) (2004) (Part I), in press.

[9] Y.L. Bai, M.F. Xia, F.J. Ke, H.L. Li, Damage field equation and criterion for damage localization, in: R. Wang (Ed.), Rheology of Bodies with Defects, Kulwer Academic Publishers, Dordrecht, 1998, pp. 55-66.

[10] M.F. Xia, Z.Q. Song, J.B. Xu, K.H. Zhao, Y.L. Bai, Sample-specific behavior in failure models of disordered media, Commun. Theor. Phys. 25 (1996) 49-54.

[11] N.G. Liang, Q.J. Liu, J. Li, T. Song, A chain network model simulating macro mechanical behavior and micro damage evolution of in-situ reinforced ceramics, in: T. Abe, T. Tsuta (Eds.), Advances in Engineering Plasticity and its Applications, Pergamon, 1996, pp. 141147.

[12] P.Y. Sheng, K. Alzebdeh, Spring network models in elasticity and fracture of composites and polycrystals, Comput. Mater. Sci. 7 (1\&2) (1996) 82-93. 